\title{
Rotational atherectomy for the treatment of isolated femoral artery traumatic lesion: a case report
}

\section{Aterectomia rotazionale per il trattamento di una lesione traumatica isolata dell'arteria femorale: un caso clinico}

\author{
Giovanni Esposito, Luigi Di Serafino, Giuseppe Gargiulo, Anna Sannino, \\ Gabriele Giacomo Schiattarella, Anna Franzone, Cinzia Perrino, Massimo Chiariello
}

\begin{abstract}
Rotational atherectomy for the treatment of isolated femoral artery traumatic lesion: a case report. G. Esposito, L. Di Serafino, G. Gargiulo, A. Sannino, G.G. Schiattarella, A. Franzone, C. Perrino, M. Chiariello.

We describe the case of a 50-year-old man with an isolated plaque of the left distal superficial femoral artery (SFA), probably not related to atherosclerosis, but rather to a traumatic event. He was admitted to our hospital because of intermittent claudication.

The critical distal SFA stenosis was documented by angiography and the lesion was treated by rotational
\end{abstract}

\begin{abstract}
atherectomy without stent implantation. At 1-year follow up, Doppler Ultrasound scan demonstrated a normal flow pattern of the left SFA and downstream districts in the absence of any complication. Therefore, rotational atherectomy is a safe and effective technique particularly in cases of peripheral arterial disease wherein stent implantation is dangerous.
\end{abstract}

Keywords: claudicatio intermittens, trauma, rotational atherectomy.

Monaldi Arch Chest Dis 2009; 72: 148-151.

Division of Cardiology, Federico II University, Naples, Italy.

Corresponding author: Giovanni Esposito MD, PhD; Division of Cardiology; Federico II University; Via Pansini 5; I-80131 Naples; Italy; Tel: +39081 746 2216; Fax: +39 081746 2223; E-mail address: espogiov@ unina.it

\section{Introduction}

Peripheral arterial disease (PAD) is characterized by a reduced blood supply to the lower or upper extremities. It is most commonly caused by atherosclerosis, but it may also result from thrombosis, embolism, vasculitis or fibromuscular dysplasia [1]. The cardinal symptom of PAD is intermittent claudication, present in approximately $20 \%$ of the population over age 70 [2].

Here we report the case of a patient presenting with intermittent claudication due to an isolated obstruction of left distal superficial femoral artery (SFA). Interestingly, the patient had no cardiovascular risk factors and no previous history of coronary artery disease (CAD), suggesting that his symptoms were not caused by atherosclerotic disease. Since he was a soccer player and reported a previous traumatic event on his left knee, we hypothesized that his focal SFA stenosis could be related to this trauma. The patient was classified as TASC IIA (Intersociety consensus for the management of PAD IIA) because of the angiographic aspect; hence, the endovascular therapy was the treatment of choice. Although percutaneous angioplasty with stenting represents the recommended strategy for this kind of lesions [3], we performed a rotational atherectomy in order to prevent stenting complications related to this district, such as stent fracture [4].

\section{Case report}

A 50-year-old man was admitted to our catheterization laboratory (Cath-Lab) because of disabling intermittent claudication and duplex ultrasound evidence of left distal SFA critical stenosis. At the time of presentation, he complained pain, numbness with mild exercise, and reduced walking capacity (maximum walking distance less than $200 \mathrm{~m}$ ) progressively worsened in the past 3 months. On physical examination his left lower limb was not pale and cool. His left femoral pulse was present but the left popliteal and left lower extremity pulses were reduced. The ankle-brachial index was 0.72 for the left limb. Right femoral, popliteal and distal pulses were present. There was no ulcer formation related to occlusive arterial disease on his legs. Moreover, the patient had no history of hypertension, dyslipidemia, diabetes mellitus, smoking and cardiovascular disease. Interestingly, the patient reported a severe knee trauma during a soccer match 3 months before the claudication started. According to his symptoms and clinical signs, he was assigned to the IIB Fontaine's stage and a diagnostic peripheral arteriography was performed. This latter revealed a focal isolated $90 \%$ stenosis in the distal SFA in the absence of other lesions in both limbs (Figure $1 \mathrm{~A})$. After heparin infusion, the procedure was performed using the contralateral approach through a 7F long sheath. Arteriography was performed initially to determine the extent 
A.

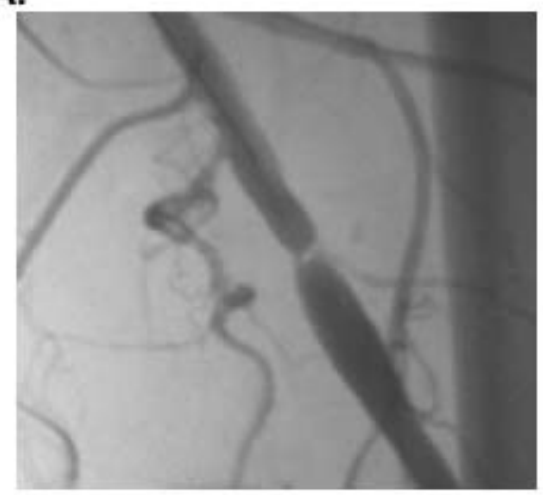

B.

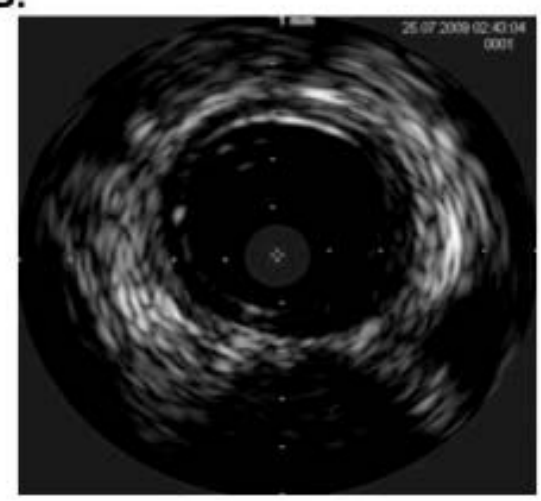

C.

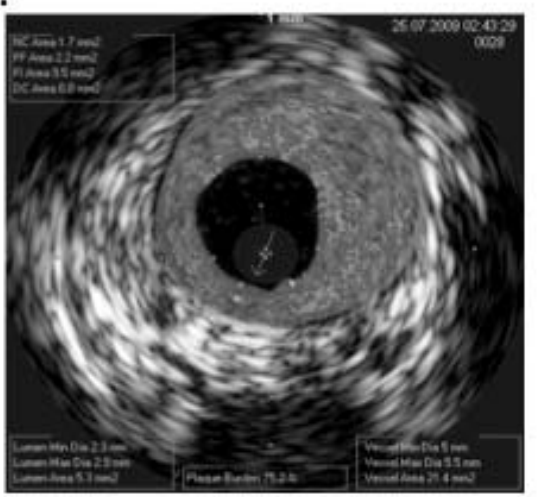

D.

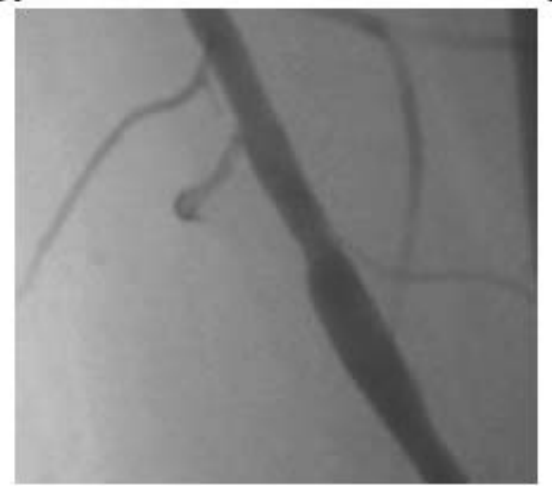

E.

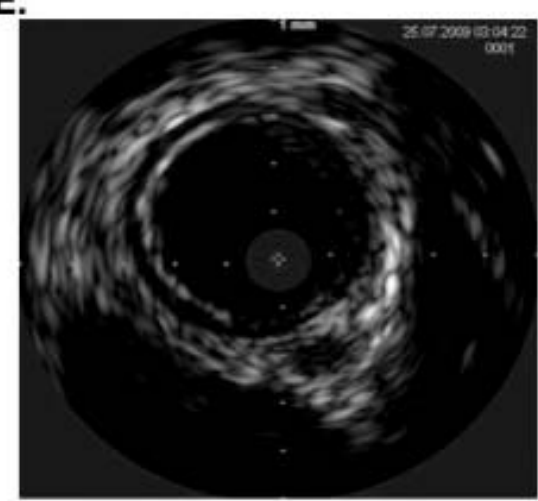

F.

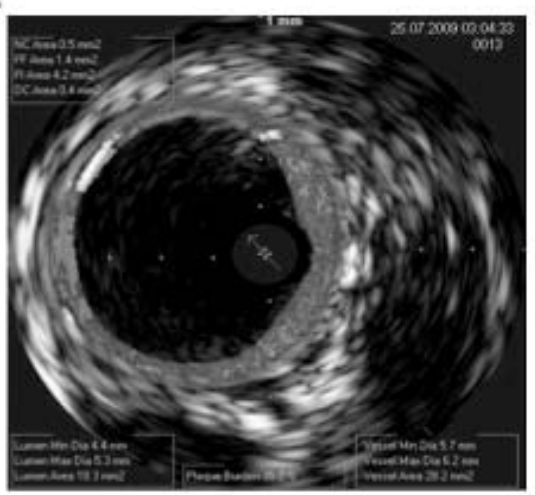

Figure 1. - A. The peripheral angiography demonstrated the occlusion of left distal superficial femoral artery (SFA). B-C. IVUS of the plaque before the rotational atherectomy. D. At the end of the procedure, excellent vessel patency was achieved with optimal angiographic result. E-F. IVUS image of the plaque after the rotational atherectomy.

of the lesion and intravascular ultrasound (IVUS) was used to better evaluate plaque features (Figure $1, \mathrm{~B}$ and C). Thus, a 0.014 " $\mathrm{BHW}$ wire $(300 \mathrm{~cm})$ was used to cross the lesion and to give support to the SilverHawk plaque excision system. The SilverHawk device (Ev3, Plymouth, Minnesota, USA) has been selected in consideration of the size of the vessel. Considering the distal femoral location of the lesion, we used a MS-M $6 \mathrm{~cm}$ Tip device. After atherectomy, both the contrast injection (Figure $1 \mathrm{D}$ ) and the IVUS images (Figure 1, E and F) evidenced an optimal result and good run-off to the patent vessels below the knee. The histological analysis performed on the plaque after the excision, allowed us to describe its composition. The ematoxylineosin images demonstrated a prevalence of fibrotic tissue with some fibroblasts and smooth muscle cells nuclei, in the absence of inflammatory cells and atherosclerosis (Figure $2 \mathrm{~A}$ ). These data were also confirmed by the positive actin immunohistochemical staining (Figure $2 \mathrm{~B}$ ). Thus, these
A.
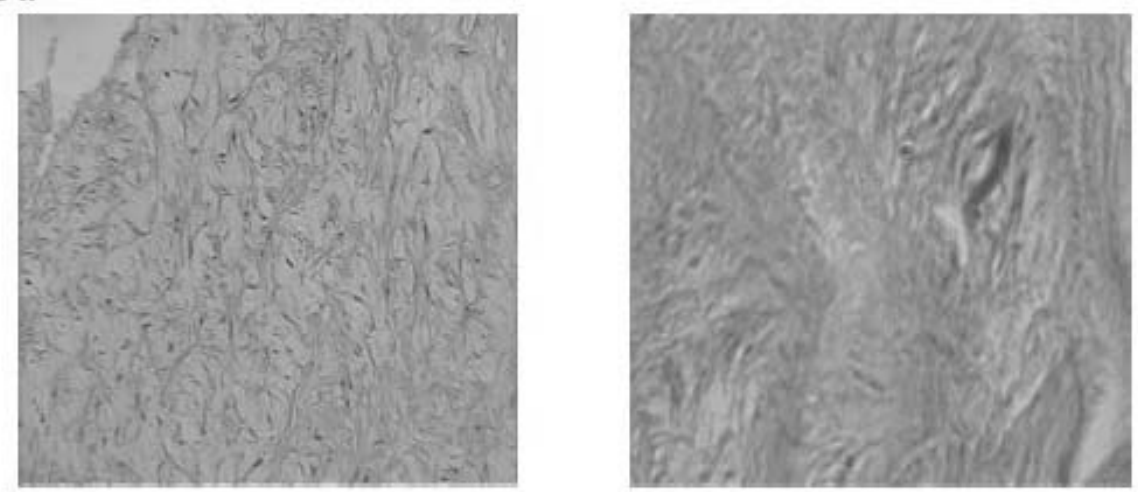

B.
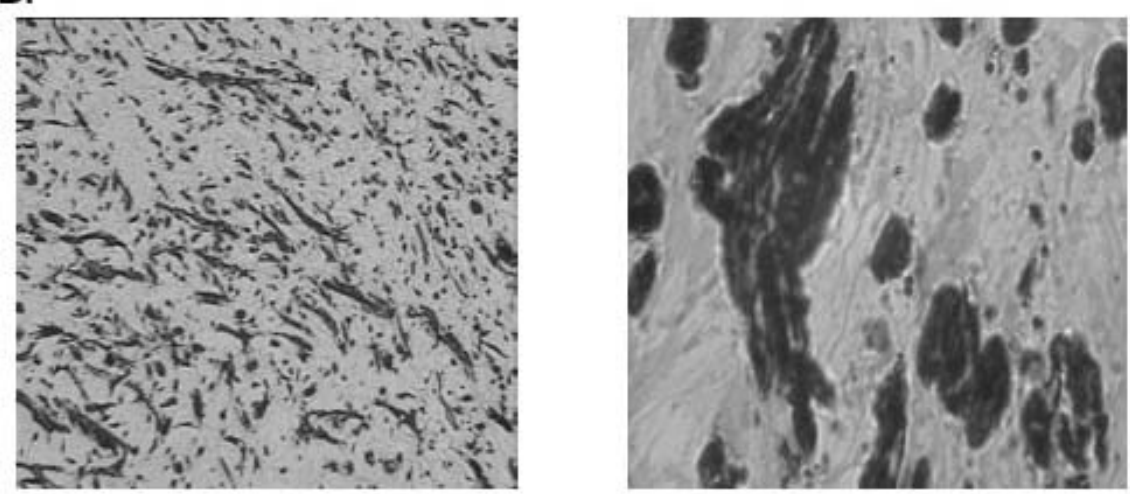

Figure 2. - A. Ematoxilin-eosin staining of the plaque section, representing its dominant fibrous composition (left: 10x magnification; right: 100x magnification). B. Actin immunoistochemical analysis resulted markedly positive (brown), confirming the fibrous composition of the plaque. 
features not only excluded an atherosclerotic origin of the lesion, but also indicated a probable post-traumatic nature. In fact, the explanted tissue was similar to the typical post-traumatic lesion, neointimal formation, observed after percutaneous transluminal angioplasty (PTA).

The patient received a load-dose of Clopidogrel $(300 \mathrm{mg}) \mathrm{x}$ os and he was discharged on dual antiplatelet therapy (ASA $100 \mathrm{mg} /$ die plus Clopidogrel $75 \mathrm{mg} /$ die). Two days after, duplex ultrasound scan evidenced a normal flow in the treated and downstream segments. At 2 months follow-up, duplex ultrasound scan confirmed a normal flow in these segments. The patient referred an improvement of walking capacity and the disappearance of pain and numbness during exercise. The left lower extremity pulses increased intensity and the anklebrachial index improved to 0.95. At twelve months follow-up, colour Doppler ultrasound demonstrated patent femoropopliteal segment and normal flow pattern.

\section{Discussion}

Atherosclerosis is the main cause of PAD. Several cardiovascular risk factors, in particular hypertension, dyslipidemia, smoking and diabetes mellitus are involved in its beginning and progression. This pathological process is characterized by lipid accumulation and mechanisms of inflammation, leading to plaques formation and progression in several vascular districts. Interestingly, we present the case of a patient without the above mentioned risk factors or any background history of CAD.

Surprisingly, the lesion determining the intermittent claudication was isolated, in fact peripheral angiography confirmed the focal distal SFA stenosis excluding any other lesion in both legs (Figure $1 \mathrm{~A}$ ). Since IVUS and histological analysis confirmed that the plaque had a dominant fibrous composition (Figures 1, B and C, and 2), and since the lesion was located close to the knee, we hypothesized that its formation was due to a traumatic event. Taken together, the sportive activity of the patient, the severe knee trauma reported few months before the symptoms started, and the histological characteristics of the plaque support our hypothesis of traumatic origin of the SFA lesion.

Given the high morbidity and mortality of PAD, different treatment options have been proposed, including femoropopliteal bypass surgery, combined thrombo-endo-arterectomy techniques and percutaneous revascularization. The indications for this last option have been extended, because of its lower invasiveness and morbidity. In fact, in the past, it was used only for short stenosis and occlusions, while, nowadays, also longer lesions are eligible for this treatment [5].

As recommended by the TASC II guidelines [6], the treatment of choice for a single stenosis $\leq 10 \mathrm{~cm}$ in length or single occlusions $\leq 5 \mathrm{~cm}$ in length (femoropopliteal type A lesions) is the endovascular therapy. The conventional endovascular therapy used for PAD is characterized by PTA and stent implantation. In fact, there is a general agreement that for acute failure of PTA of a SFA lesion, stent placement is indicated [6]. Moreover, a recent randomized trial has demonstrated significantly higher primary patency rates of stenting vs. PTA of femoropopliteal artery lesions TASC A and B at 1year follow up [7]. However, several alternative endovascular options are available, including subintimal angioplasty, cryoplasty, cutting balloon angioplasty, primary as well as adjunctive deployment of self expanding bare nitinol or covered stents, and ablative modalities such as excimer laser and directional plaque excision [3]. The long term results of these techniques are currently unknown.

PTA stenting for revascularization of the SFA achieves high success rates, however late clinical failure due to in-stent restenosis, clinical deterioration and stent fractures remain a major concern $[4$, $8]$. In the present case we report the use of rotational atherectomy, instead of the common PTA stenting, to treat a distal SFA lesion. Given the possible traumatic nature and, most of all, the site of the patient's lesion (distal SFA), we decided to perform a rotational atherectomy. In fact, external stent compression in this district may cause restenosis and also stent erosion, and finally fracture when it occurs repeatedly [4]. In contrast, atherectomy offers the theoretical advantages of eliminating stretch injury on arterial walls and reducing the rate of restenosis [3]. In addiction, it removes the obstructing atherosclerotic or intimal hyperplastic lesions without the disadvantage of a foreign body such as a stent in the artery [9]. The plaque excision offers also the opportunity to perform molecular or histological analyses on the sample. If re-intervention is required after atherectomy, this can be generally performed at the same site with low risks of complications or discomfort for the patient. Finally, atherectomy, in contrast to stenting, does not exclude the use of bypass surgery nor changes the anastomotic sites if surgical bypass is necessary [9].

We performed a 1-year follow up in which repeated ultrasound scans demonstrated the patency of the femoropopliteal segment and normal flow pattern. None of the typical complications of percutaneous procedures (thrombosis and emboli) or atherectomy in particular (vessel wall perforation, creation of arteriovenous fistula) [10] occurred.

In conclusion, the most striking features of our report are the potential for existence of non-atherosclerotic post-traumatic SFA lesions and the efficacy and safety of rotational atherectomy as an alternative to PTA and stenting.

\section{Riassunto}

Riportiamo il caso di un uomo di 50 anni che giungeva alla nostra osservazione per riferita claudicatio intermittens. L'esame angiografico ha evidenziato una lesione critica, isolata, della arteria femorale superficiale (SFA) distale, in assenza di significativa patologia aterosclerotica a carico dei restanti distretti. La lesione è stata trattata mediante aterectomia rotazionale senza l'impianto di stent. Ad 1 anno di follow-up, l'esame eco-doppler ha dimostrato un flusso normale sia nella SFA che 
nei distretti a valle. Pertanto, possiamo ritenere che l'aterectomia rotazionale è una tecnica sicura ed efficace nel trattamento di occlusioni vascolari periferiche in tutti quei casi in cui l'impianto di stent è controindicato.

\section{ABBREVIATIONS LIST:}

ASA: Aspirin

CAD: Coronary Artery Disease

Cath-Lab: Catheterization Laboratory

IVUS: Intra Vascular Ultra Sound

PAD: Peripheral Arterial Disease

PTA: Percutaneous Angioplasty

SFA: Superficial Femoral Artery

TASC: Inter-society consensus for the management of PAD

\section{References}

1. Libby B, Mann, Zipes. ed Braunwald's heart disease: A textbook of cardiovascular medicine. Eighth edition. 2007.

2 Criqui MH, Denenberg JO, Langer RD, Fronek A. The epidemiology of peripheral arterial disease: Importance of identifying the population at risk. Vasc Med 1997; 2: 221-226.
3 Garcia LA, Lyden SP. Atherectomy for infrainguinal peripheral artery disease. J Endovasc Ther 2009; 16: II105-115.

4 Babalik E, Gulbaran M, Gurmen T, Ozturk S. Fracture of popliteal artery stents. Circ J 2003; 67: 643-645.

5 Scheinert D, Laird JR, Jr., Schroder M, Steinkamp H, Balzer JO, Biamino G. Excimer laser-assisted recanalization of long, chronic superficial femoral artery occlusions. J Endovasc Ther 2001; 8: 156-166.

6 Norgren L., Hlatt WR, Dormandy JA, et al. Inter-society consensus for the management of peripheral arterial disease (TASC II), Eur J Vasc Endovasc Surg 2007; 33 (S1): S1-75 Epab 2006 nov 29.

7 Schillinger M, Sabeti S, Loewe C, et al. Balloon angioplasty versus implantation of nitinol stents in the superficial femoral artery. N Engl J Med 2006; 354: 1879-1888.

8 Schlager O, Dick P, Sabeti S, et al. Long-segment sfa stenting-the dark sides: In-stent restenosis, clinical deterioration, and stent fractures. J Endovasc Ther 2005; 12: 676-684.

9 Shrikhande GV, McKinsey JF. Use and abuse of atherectomy: Where should it be used? Semin Vasc Surg 2008; 21: 204-209.

10 Lin S, McKinsey JF. Plaque excision for the treatment of infrainguinal arterial occlusive disease. Tech Vasc Interv Radiol 2005; 8: 165-168. 